\title{
Applications of RFID technology in dismounted soldier solution systems - study of mCOP system capabilities
}

\author{
Mariusz Chmielewski ${ }^{1, a}$, Marcin Kukiełka ${ }^{1}$ \\ ${ }^{1}$ Military University of Technology, Cybernetics Faculty, gen. S. Kaliski Street, Warsaw, Poland
}

\begin{abstract}
This paper discusses application of RFID technology in Dismounted Soldier Solutions gathered from the development and demonstration of mCOP platform. The software has been developed to elaborate Network Enabled Capabilities in tactical environments. Presented research was a part of demonstration prepared for the European Defence Agency. The main aim of the tool is the delivery of command support capabilities utilizing data fusion and integration mechanisms implemented in heterogeneous military mobile networks. Application of military integration standards such as JC3IEDM, NFFI and TSO supports interoperability with BMS class systems. Utilisation of RFID technology in mCOP software has several purposes, mainly used for process automation of authorisation and personal identification. Indirectly such functionalities can be used for implementation of UAV supported - Blue Force Tracking and IFF services. This research elaborates a set of implemented ideas for such solutions. Developed system serves as a multi-level and multi-platform command and control system supporting military and crisis operations. A crucial part of the system - mCOP, the handheld application for tactical level commanders, serves as personal toolkit for location monitoring and combat situation distribution and management. The tool delivers a set of tailored functionalities supporting commander's situation awareness and decision making process. The mCOP hosting device equipped with RFID delivers also means of tactical, emergency, micro network communication.
\end{abstract}

\section{Introduction and Main Concept}

The main concept which has been driving the development of mCOP is to utilise commonly available mobile platforms and equip them with secure, fully functional application supporting main activities during combat missions. Military operations in $21^{\text {st }}$ century require more and more information technology support in order to share information and decision superiority. Deployment of C4ISR systems in military has been conducted for almost three decades and the availability of smaller, smarter and more powerful handheld devices provides new opportunities for ICT tools. The deployment is constantly progressing moving downwards from operational unit command centres to tactical level command posts and further to task groups and even individual soldier. Such solutions (Dismounted Soldier Solutions) do not provide established, referenced functionalities as they represent research projects rather than deployed solutions. Also, the majority of them concentrate on integration of weapon systems rather than command and decision support or location and biomedical monitoring.

Described solution mCOP is a part of larger iCOP distributed C2 platform, delivered in form of a SOA environment available through stateful web portal. The technology used for implementation, was one of the crucial aspects tested in the research. The preferably more valuable part of research is connected with the design and composition of functionalities for mobile personal application, carried by task force members and utilised directly on the tactical level.

The availability of handheld devices and their usage during combat is often considered as a supplementary form of supporting soldiers and commanders. Especially in case of a crisis situations where any form of communication, monitoring, data sharing can be a valuable issue. Such observation was the base assumption for mCOP application, which should be treated as a tool for data fusion and integration but mainly the production of soldier's situation awareness during operation.

The security issues connected with processing of classified or potentially sensitive data, were one of the problems considered during architecture design and software development phase. Many of such problems could not be addressed due to many features of the devices such as utilisation of commercially available hardware platforms and operating systems. However, conducted research is aimed at utilising any available computing means, which can be used to help and support individuals during combat or crisis, at the same time maintaining acceptable security levels. Such compromise rises from the main assumption: deliver software, easily installable which can be used as a portable combat support tool. The ideal case assumes that any member of

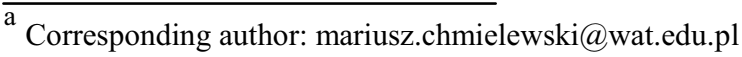


a task group is able to acquire and configure the mCOP application on his personal or tactical handheld. To simplify configuration a RFID technology has been applied, which serves as remote identification mechanism. Using RFID soldier can $\log$ in to C2 system and preconfigure all GIS and mission data. RFID technology simplifies also integration with the logistics system/module where user obtains combat gear, ammunition and communication equipment. Depending on C2 system's The configuration and registration automation features are able to shorten TSU (Tactical Small Unit) combat preparation and departure time by almost 30\%.

\section{Concepts and features of Dismounted Soldier Solutions}

A Dismounted Soldier Solution is a class of system going beyond only a software platform, as it multi-layer, wirelessly interconnected computer system integrated with weapons and specialised observation and reconnaissance gear. A valuable summary of such systems has been presented in [14]. The definition itself has not yet been unified and systems such as Land Warrior [15], ISW Tytan [14], IdZ-ES [14], FIST [17], FÉLIN [16] and many more, has been developed as technology demonstrators and testbeds for researching operational capabilities. Going into design details each and every system has been aimed at increasing higher combat effectiveness of the individual and by application of NEC concepts the unit itself. However, different systems emphasise various functional elements such as weapon system, camouflage capabilities, survivability, observation and sight equipment. For the purpose of this work we will discuss mainly ICT related capabilities of such solutions as the innovation and technology opportunity lies not in the tactical gear itself but extending the features of that gear with computer technology. In case of NEC concept implementation in DSS systems aim mainly at time constrained data distribution, sensor data utilisation and combat information sharing. The development of technology and processing power delivered new types of intelligent sensors and equipment, which in conjunction with wireless integration mechanisms and large data processing power give new opportunities for mobile platforms usage in combat.

The iCOP platform and mainly mCOP mobile application addresses rather different approach to software utilisation in combat, concentrating on functionalities mainly connected with soldier's situation awareness, decision support and mission guidance.

Available DSS systems maximise data sharing functions interconnecting soldiers within TSU and delivering:

- integration with command C4ISR systems,

- combat situation distribution, ${ }^{*}$

- voice communications,

- tactical picture or recon video sharing,

-reconnaissance and surveillance information delivery,
- mission report notifications,

- location monitoring

- GIS information delivery.

Such functions offer preliminary set of information required during combat especially in dynamic asymmetric conflict environment. However, soldiers may require additional set of services, which will assist them in three stages of operation:

- the briefing and departure,

- execution,

- post-reporting.

The requirements on each and every phase should support the soldier or commander and simplify activities indirectly connected with the mission itself. One of the crucial features of DSS system is to facilitate data sharing while delivering only useful and eligible information.

\section{Situation awareness as a DSS -mCOP implemention [18]}

Situation Awareness (SA) has been defined in [1] as "the perception of the elements in the environment within a volume on time and space, the comprehension of their meaning and projection of their status in near future" and is now being a subject of study in a number of domains $[2,7,12]$. This concept is a key feature around which presented work has been organised. mCOP system utilizes handhelds at the lowest levels of command for the development of near real-time tactical picture and soldier's situation awareness.

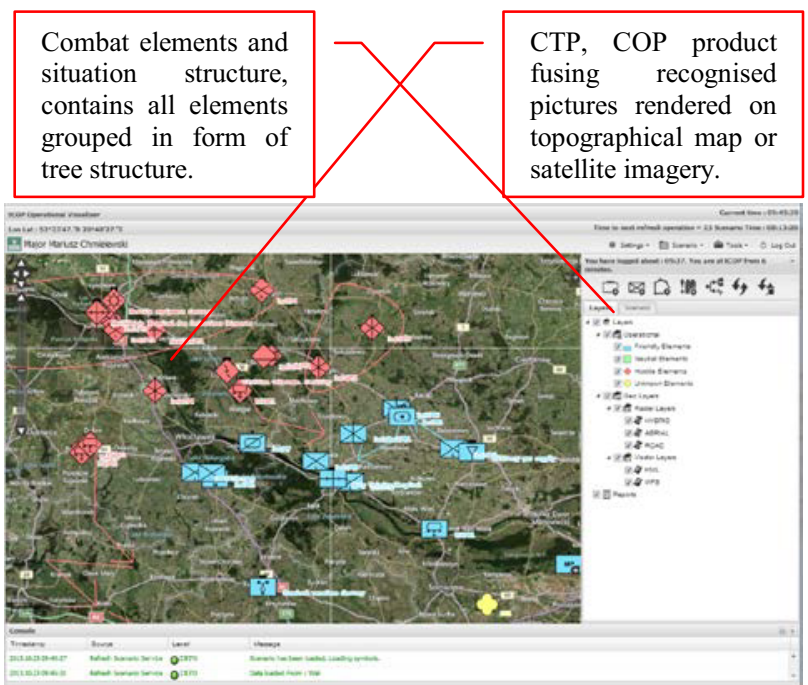

Fig. 1. tCOP battalion level operational picture fused from all subordinate level units and supplemented with C4ISR decision recommendation

The mCOP application automates many monitoring activities such as geo-location, intensity of activity, speed, bearing and even vital functions of a commander or a soldier. The process consists of two concurrent activities. The first one is aimed at the battlefield situation's data acquisition and delivery. The data provided by the sensors and obtained from the subordinates is derived and filtered on the handheld device itself and then sent to the tCOP server for further 
processing. The data describes the movement parameters of a given unit based on the fused data from the unit's staff. The second activity is oriented at acquisition of the battlefield situation's data from the server. The tCOP server obtains data from the automatized processes as well as from the template-based messages and other sources. The tCOP server filters collected data to match the situation in the vicinity of the user. Then the data is sent to the mCOP devices via sustained feedback connection.

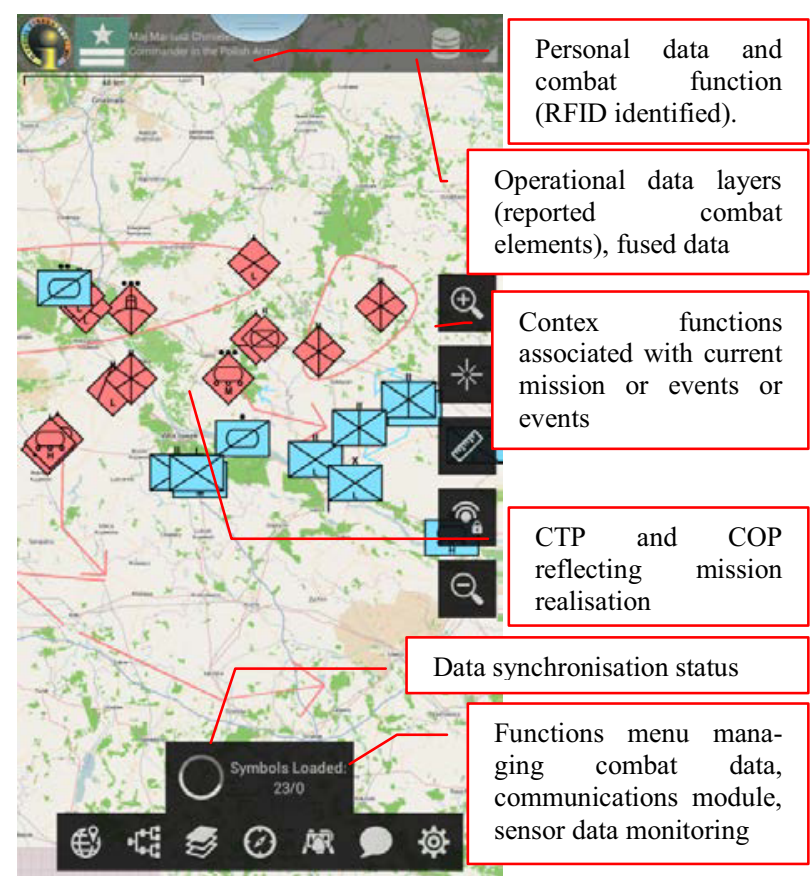

Fig. 2. mCOP main CTP, COP view containing - fused data and GIS information

The enrichment of users' $\mathrm{SA}$ is achieved by presenting inertial sensor data and using Augmented Reality perspectives containing the operational information. For SA development we combined common handheld sensors' data such as GPS, magnetometer, accelerometer, and gyroscope. Furthermore mCOP delivers efficient route planning capabilities and combat scenario simulation assessment.

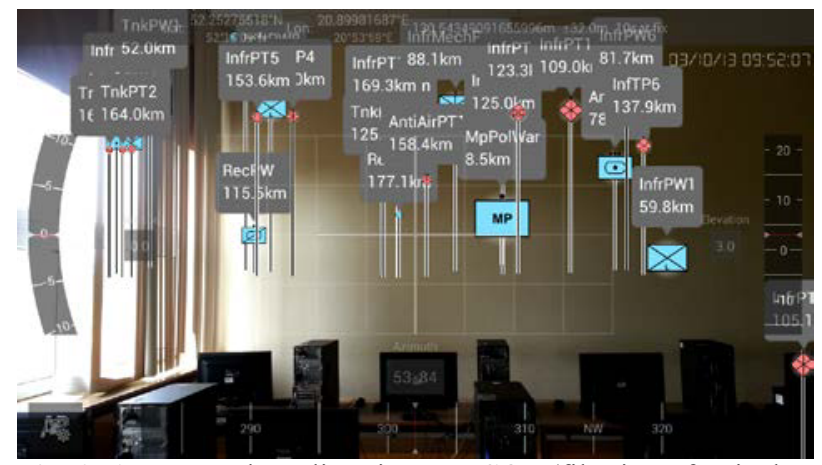

Fig. 3. Augmented Reality view - mCOP (filtering of units has been disabled thus rendering all reported elements)

To enrich the communication capabilities within the tactical network mCOP and tCOP provides secure and reliable template-based ADat-P3 military messaging.
Military operations are conducted mostly in difficult conditions in terms of the environment and the information. In such situation the lack of visibility and proper orientation locating and identifying enemy and friendly forces may be-come problematic; better situational awareness is required for effective operation. Believing that situational awareness in these circumstances cannot be met by using traditional approaches we decided to research Augmented Reality View.

The Augmented Reality view provides the data fusion products in form of one picture on which the application evaluates the positions of allied and enemy forces within the range of operation supplemented with the current observer location and device orientation. This information can be further semantically pro-cessed to recommend a commander the most efficient course of action or movement route. Augmented Reality is a construct built using data about battle-field, location, data derived from sensor fusion and above all the camera view. To create the AR View there is a need to describe the vertical and horizontal field of the camera's view. This is done by measuring the visible angle one meter away from the camera. Specifying the angles and maximum distance to the shown units allows further view creation.

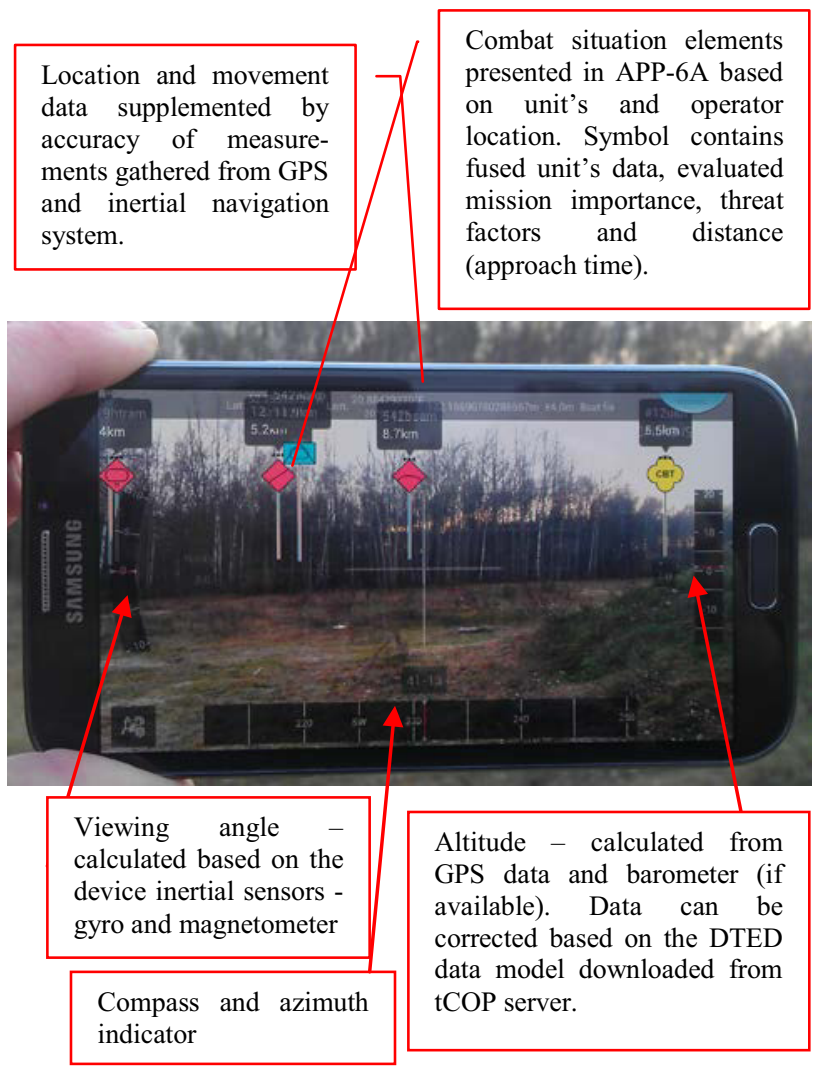

Fig. 4. Augmented Reality view rendering elements from CTP and COP products and viewing situation actors and evaluated SA characteristics

Implemented rendering mechanisms determines if the object should be shown on the screen and how to show the icons expressed in tactical symbology, evaluating their locations, azimuth, distance, and most of all mission importance and threat status (Fig. 3, 4). 


\section{RFID technology area of application}

RFID $[19,20]$ technology is well recognised standard for secure, wireless, remote identification and tagged objects tracking. In crisis operations such features can be utilised in many areas such as casualty identification, equipment or object tracking, combat gear configuration and assembly. Presented research discusses utilisation of this technology for handhelds supporting combat missions.

The mCOP application utilises RFID based features in three areas personal data identification, near field communications (serving as a backup emergency channel) and prototype IFF (Identification Friendly Foe) and BFT (Blue Force Tracking) services performed by assisting UAVs.

RFID in any DSS solution can be used to configure personal handheld which represents individual (using unique identifier and stored profile) which can be utilised for information access and crucial during medical assistance especially in the combat zone. The mCOP implementation solves time consuming authentication (perimeter control) and configuration issues during mission preparation and execution. The key advantage in such scenarios is connected with the simplification of tasks leading to mission preparation. The automation of such activities relief the operators and help them concentrate directly on the mission itself.

The mCOP toolkit utilising RFID mechanisms and functionality simplifies the departure and configuration phase by automation of soldier identity and credential verification. Using handheld with RFID or NFC technology user confirms the equipment and weapons acceptance for the mission, during which the logistics system checks if the equipment configuration is correct. Such functionality can be extended towards not only personal equipment but also vehicles. System supports also distribution of final forces and equipment TSU setup which normally is reported manually.

There are also application areas where RFID can be used dually as an emergency communication channel. Based on the near-field features of the RFID radio the mCOP can utilise such peer-to-peer channel for data transmission. Employing specialised algorithm and mechanisms for data aggregation and verification we can obtain means of communication on the lowest levels during radio-interference. For such case mCOP offers optimised tactical chat communications enabling dedicated means of communicating within TSU.

RFID can increase the efficiency of operation by providing each soldier in the squad with a tool for digital identity carrier and reader. Applying correctly configured RFID modules and utilising tactical UAV's, we can obtain which individuals are members of friendly forces which can be a form of simplified personal IFF service. To achieve such functionality a set of algorithms of location estimation must be developed and a specialised AR viewer functionality delivered.

Despite blue force tracking issue there are several areas in which RFID implementation can improve significant processes or even provide additional battlefield information.

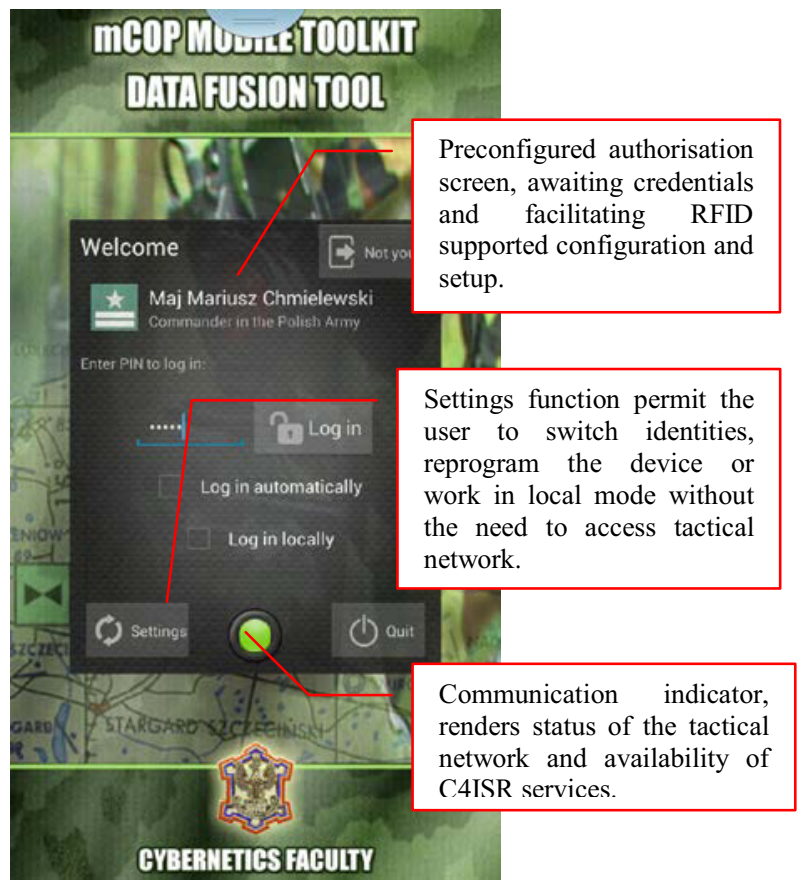

Fig. $5 \mathrm{mCOP}$ authorisation and preliminary configuration screen rendered after device restart or mCOP activation.



Fig. 6 Tactical chat functionality utilising any communication channel including tactical IP network, GSM, WiFi Direct and RFID based data sharing within TSU.

\section{Battlespace data fusion and integration}

Creating advanced sensor based views requires fusing the sensors output in order to obtain a reliable 
information. In created solution data from inertial sensors and magnetometer are utilized to obtain the position of the handheld in 3-dimensional space described by the Euler angles, pitch, roll and yaw, with yaw being nothing else than the azimuth. Moreover these angles have to be appropriate despite of the orientation of the phone, meaning that the coordinates system is changing along with orientation of the device. When the device is held flat the azimuth is based on the direction that the top of the device is pointing. To smoothen the sensor data a low-pass filter has been applied.

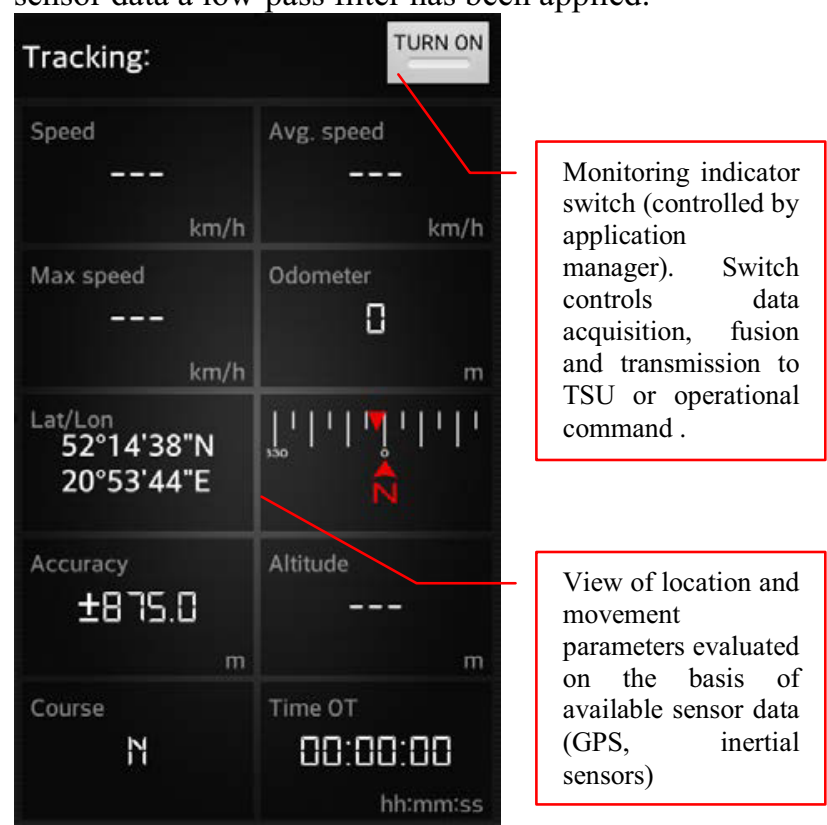

Fig. 7. mCOP monitored location and movement data, collected and retransmitted during mission execution. .

The fact that the operational data is often supplied from various, heterogeneous sources causes many integration problems on technical, syntax and semantics level. Implemented data fusion process provides data association and state estimation methods. Data processed in such way are more reliable and accurate which is significant in terms of developing multiresolution operational picture for crisis management support.

Data association is commonly used in cluttered environments (meaning that targets are close to one another). These situations hinder tracking targets and accurately determine their position. It is worth mentioning that data association is the key element due to higher levels of fusion process, like e.g. state estimation, which leads to incorrect behaviour if data association is incoherent. In order to improve the reliability of developed environment, we used three data association techniques and algorithms: nearest-neighbour algorithm, K-means algorithm and probabilistic data association including credibility factor. Obtained results are passed through majority voting mechanism. This approach reduces disadvantages of separately used methods providing reliable and accurate data association.

State estimation techniques have been used in order to determine, a state of the target especially while moving. The ultimate goal of these methods is to obtain a global state of the target basing on the given observations. During the revision of available sources for conducting an estimation process, one of most flexible methods has been applied - the particle filter. They cope successfully with both non-linear dependences and nonGaussian density functions in dynamic systems and noise. Nevertheless, in order to obtain low variance, the filter needs huge quantities of data, which comes at the cost of computational power. To reduce the amount of particles a significant assumption has been made tracking of the targets is processed individually with an assumption that they do not interfere with any particles. Estimated state of the target enriches the COP product and provides additional information to support a decision maker. In developed prototype this method is used mainly to estimate the route of the object and determine its combat potential.

Utilised heterogeneous data sources deliver data, which can be considered to be insignificant or invalid. We implemented methods which extract the important data by utilizing fusion techniques. Such algorithms support the reliability and accuracy of the Common Operational Picture and situation awareness [3] development thus improving decision support tools. An important aspect of the system is its data integration and acquisition capabilities. Such features in tCOP have been implemented as NFFI standard services and JC3IEDM compliant database.

NFFI (NATO Friendly Force Information) [4] is an interoperability standard initiated in 2005 by Allied Command Transformation (ACT) in response to an urgent need to exchange friendly force tracks between national Command and Control Information System (C2IS) within the ISAF coalition. TSO (Tactical Situation Object) [5] defines standard for data representation and a semantic model of a situation representation for crisis management and dispatching system perceived by a particular observer at a particular time. It is utilised to transfer data from such a view to console system or a dispatcher.

\section{Architecture and implementation technology stack}

The rendering of a COP utilizes data fusion algorithms and integration connectors. The major functionality of any military decision support system is GIS referenced data and functionalities for reflecting current or planned operational situation expressed in tactical symbology (APP-6A or country specific standard). It represents combat scenarios in form of pure relational data requires additional algorithms to present the situation picture, which can further be reused by the decision makers [7]. These tools enable allied (NATO) commanders to increase their Situation Awareness (SAW), which in case of our system can be understood as a perception of battlespace environment within a volume of time and space, and the projection of their status in the near future [8]. Such an approach enables collaboration between environment elements, improves synchronization and reduces delays of the communication. mCOP simplifies the delivery of CTP and COP to every soldier in that particular military unit. Usage of smartphones and 
wireless tactical networks improves the availability of toolkit. Services provided by the tCOP command system provides current situation including both military and civilian objects, information concerning both in Area of Responsibility - AOR and in Area of Interest - AOI. All requirements for COP and CTP products have been described in [9].

After review and analysis of set of available technologies on the market has been selected, fulfilling requirements for GIS tools with operational symbology editor capabilities and a stateful GUI, delivered through a browser. The tCOP a command centre application has been developed as a multilayer Java Enterprise system supplemented with GWT and Sencha GXT libraries facilitating GIS user interface. The choice of technology stack has been made based on the availability of open source libraries, flexibility of extensions and vendorneutral characteristics. tCOP has been designed and developed according to multi layered architecture so as to allocate the different responsibilities of software to different layers. Client side has been developed according to the concept of RIA (Rich Internet Application). This model utilizes desktop application approach delivered as AJAX based client components executed in any web browser. GWT (Google Web Toolkit) is a set of tools and components supporting the development of RIA applications in Java language. The resulting GWT application consists of client side layer HTML and JavaScript generated by GWT transformation engine from Java code and set of server side services (mostly asynchronous). The framework provides support for communication with server using GWT-RPC mechanism wrapped for AJAX calls from client to server side, providing stateful user interface and rapid application delivery.

Security characteristics in case of combat support application is one of crucial elements, especially when software is deployed on commercial equipment. Server side utilizes security layer consisting of authentication and authorization services for mobile application functionalities as well as for integration services responsible for data exchange and integration. The security layer utilizes also SSO (Single sign-on) infrastructure to simplify distributed and multiplatform authentication. Such approach in conjunction with RFID technology reduces time needed for logging and registering with-in the tactical network and simplifies user access within military C4ISR systems domain.

Business logic layer consists of SOA based services implemented using Enterprise Java Beans (EJB 3.1) components. tCOP web client layer backend software communicates with EJB's using Business Delegate object's implemented over HTTP standard: JAX-WS web services and GWT-RPC servlets.

Persistence layer have been implemented using Java Persistence API (JPA 2.1) standard approach for Object to Relational Mapping (ORM) as well as EclipseLink, which has been used for both management and auditing of data.

The solution provides extensive GIS mechanisms in form of SOA components dedicated at obtaining spatial data and maps. Moreover it is capable of rendering both raster and vector type terrain layers in the same time with possibility of setting order of them. User has ability both to use one of many predefined web mapping services' sources: OpenStreetMap, Bing, Landsat7 or any other custom web map service consistent with either WMS or WFS standards. To further expand the capabilities of the spatial data visualization, KML and GML standards have been used to describe spatial data sources.

tCOP solution allows visualization of operational situation in terms of military and civilian objects, their actions as well as equipment and supplies. It reflects the situation both by placing objects on the map and in the scenario objects widget representing formation's command chain, all objects and administrative structure. tCOP provides mechanism to visualize both military and civilian objects matching APP-6A or TSO standards. Described solution is not only a tool for visualization of current battlespace situation, but also a robust application mechanism for mission planning (adding units, infrastructures and tasks).

All data and messages are encrypted using RSA algorithm to ensure high level of security. Tactical voice or message communication in the system has been provided in form of tactical chat. To facilitate and speed up the messaging process tCOP provides messages' templates often used in both military and civilian communication.

OPresented solution iCOP provides scalable environment capable to develop and deliver COP and CTP products for the tactical and operational level commands. Crucial features of the environment deliver distributed SOA based and reliable set of integration and decision support be used as ort services, tuned for web and mobile access. The application of new technologies has been done intentionally in order to verify the Network Enabled Capabilities concepts in military tactical networks supporting civilian, commercially available technologies for dedicated security-oriented solutions.

Server-oriented tCOP is dedicated for a decision support of tactical level command. System has been developed using state of the art technologies and best practices. Supplied tools enable allied (NATO) commanders to increase their productivity of mission planning and operation execution. tCOP is one of few web-based applications used for heterogeneous data integration and Common Operational Picture development. mCOP mobile application is dedicated to the lowest levels of command, supporting efficient operational data flow and automatic monitoring of forces. The solution supports delivery of detailed battlespace information concerning objects, units, equipment and installations their combat potentials and tasks. Using the application the soldier is able to gather specific tactical information on equipment, warfare as well as unit resources and tasks. Furthermore, detailed combat information contains not only static data, but also description of unit's behavior in time (tasks). 


\section{Summary}

Presented family of mission support tools (tCOP, mCOP) provides the information infrastructure for military and civilian crisis operation support. The tools utilise means of applying new technologies to increase the efficiency of combat operations on any stage of mission execution. The tool is able to consume and produce standardised data sources in form of NFFI and JC3IEDM data messages and TSO dispatch services which delivers standardised interfaces for COP and CTP acquisition. The demonstrator have been validated in several deployment configurations utilising ad-hoc tactical networks, server environments and variety of android devices (also ruggedized). The iCOP platform is able to process and refine operational scenario elements fusing the data from many heterogeneous data sources. Application of AR to support situation awareness of TSU member delivers new means of information rendering increase of the decision making efficiency during combat exercises. Application RFID standards simplified many activities connected with mission configuration and preparation phase. A handheld device can be used as a secure identification device storing crucial personal (including medical, ER) data. The tools have been demonstrated to EDA officials. Further research will be organised in three main areas extending the decision support capabilities, RFID applications for facilitating local communications as well as IFF and BFT service implementations. Furthermore the availability of RFID technology variants e.g. NFC, gives new opportunities for dual applications especially on the handheld market.

\section{References}

1. M. Endsley, „Design and Evaluation for Situation Awareness Enhancement," w Proceedings of the Human Factors and Ergonomics Society Annual Meeting, 1998.

2. M. R. Endsley, D. J. Garland, Situation Awareness Analysis and Measurement, CRC Press, 2000.

3. M. Chmielewski, „Ontology Applications for Achieving Situation Awareness in Military Decision Support Systems," Lecture Notes in Computer Science, nr DOI: 10.1007/978-3-642-04441-0_46, pp. 528-539, 2009.

4. NATO, NATO Fiendly Force Interoperability standard, STANAG 5527.

5. CEN WS/ISDEM, Definition of the OASIS Tactical Situation Object, 2006.

6. CEN, Disaster and emergency management - Shared situation awareness - Part1: Message structure., 2009.

7. M. G. D. Endsley, Situation Awareness Analysis and Measurement: Analysis and Measurement., Lawrence Erlbaum Associates, 2000.

8. M. Chmielewski, „Data fusion based on ontology model for common operational picture using OpenMap and Jena semantic framework," w Proceedings of the Military Communications and Information Systems Conference, Cracow, 2008.

9. NATO, NATO Common Operational Picture (NCOP) - NC3A, 2006.
10. M. Chmielewski, A. Gałka, „Semantic Battlespace Data Mapping Using Tactical Symbology," Advances in Intelligent Information and Database Systems, DOI: 10.1007/978-3-642-12090-9_14, pp. 157-168, 2010.

11. W3C, Web Service Glossary, 2011.

12. ,Shared situation awareness - Part1: Message structure," CEN.

13. M. J. Zieniewicz ; D. C. Johnson ; C. Wong ; J. D. Flatt, "The Evolution of Army Wearable Computers", IEEE, Pervasive Computing, vol.1, no. 4, pp. 30-40, 2002, doi: 10.1109/ MPRV.2002. 1158276

14. K. Frącik, New technologies and means of combat shaping modern military operations, Journal of KONBiN 3(19), 2011, ISSN 1895-8281

15. N.L. Clifton Jr., D.W. Copeland, The Land Warrior Soldier System: A Case Study for the Acquisition of Soldier Systems, Monterey, California, MBA Professional Report, 2008

16. FELIN soldier system, Sagem Défense Sécurité brochure, available online, 2008.

17. FIST - Future Infantry Soldier Technology, http://www.army-technology.com/projects/fist/

18. M. Chmielewski, et.al. Development of Operational Picture in DSS Using Distributed SOA Based Environment, Tactical Networks and Handhelds; Information Systems Architecture and Technology: Selected Aspectss of Communication and Computational Systems, 2014, Wrocław, ISBN/ISSN: 978-83-7493-856-3

19. S.A.Weis, RFID (Radio Frequency Identification): Principles and Applications, 2007, MIT CSAIL

20. I. Angell, J. Kietzmann, RFID and the end of cash?. Communications of the ACM 49 (12): 90-96. 2006, doi:10.1145/1183236.1183237. 